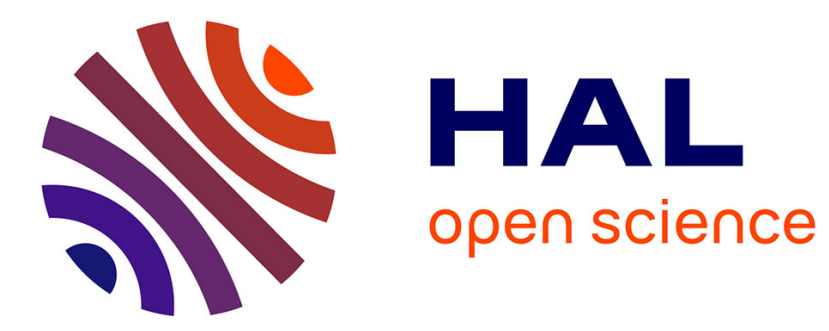

\title{
Computation of Eddy Current Losses in Soft Magnetic Composites
}

\author{
Carlo Appino, Oriano Bottauscio, Olivier de La Barrière, Fausto Fiorillo, \\ Alessandra Manzin, Carlo Ragusa
}

\section{- To cite this version:}

Carlo Appino, Oriano Bottauscio, Olivier de La Barrière, Fausto Fiorillo, Alessandra Manzin, et al.. Computation of Eddy Current Losses in Soft Magnetic Composites. IEEE Transactions on Magnetics, 2012, 48 (11), pp. 3470 - 3473. hal-00825522

\section{HAL Id: hal-00825522 \\ https://hal.science/hal-00825522}

Submitted on 23 May 2013

HAL is a multi-disciplinary open access archive for the deposit and dissemination of scientific research documents, whether they are published or not. The documents may come from teaching and research institutions in France or abroad, or from public or private research centers.
L'archive ouverte pluridisciplinaire HAL, est destinée au dépôt et à la diffusion de documents scientifiques de niveau recherche, publiés ou non, émanant des établissements d'enseignement et de recherche français ou étrangers, des laboratoires publics ou privés. 


\title{
Computation of eddy current losses in Soft Magnetic Composites
} MAGNETIC COMPOSITES (SMCs) are attractive for modern electrical machine applications. On the one hand, they display isotropic behavior, allowing the circulation of three-dimensional magnetic flux paths [1]. This is a clear advantage in modern electrical machine topologies, such as axial flux machines [2][3], or claw pole generators [4]. On the other hand, their granular structure tends to inhibit macroscopic eddy current pattern, making them suitable for high-speed machine cores [5]. In these high-frequency applications, a correct evaluation of the eddy current classical loss component is crucial.

It is generally assumed [6][7] that eddy currents in SMCs can be associated with paths either confined within the grains or extending, because of imperfect insulation between grains, over the scale of many grains, eventually investing the whole sample cross-section. As shown by the homogenization theory [8], this empirical separation of eddy currents is not straightforward from a mathematical viewpoint and the degree to which its application is justified must be clarified. It has been suggested that the measured electrical resistivity $\rho_{\text {meas }}$ of the material can be taken as a direct entry for the macroscopic loss model [6]. This approach could be justified in materials where, like in [6], the grains are well separated and the material conductivity is dictated by the conductivity of the insulating filler. However, in the practical high-density SMCs for electrical machines, the measured resistivity is chiefly the one arising from the random contacts between the grains [9]. Consequently, the link between the measured resistivity $\rho_{\text {meas }}$ and the macroscopic loss is not obvious. In Ref. [10], the authors tried to circumvent the problem by identifying equivalent deterministic contact properties from the loss measured in an SMC sample of a given cross-sectional area, resulting in a general formulation of the classical eddy current loss for samples of generic cross-sectional area. However, it would be interesting, both from a theoretical and practical point of view, to link the classical loss to the measured

\author{
C. Appino ${ }^{1}$, O. Bottauscio ${ }^{1}$, O. de la Barrière ${ }^{1,2}$, F. Fiorillo ${ }^{1}$, A. Manzin ${ }^{1}$, C. Ragusa ${ }^{3}$ \\ ${ }^{1}$ Istituto Nazionale di Ricerca Metrologica (INRIM), Torino, Italy \\ ${ }^{2}$ SATIE, ENS Cachan, CNRS, UniverSud, 61 av du President Wilson, F-94230 Cachan, France \\ ${ }^{3}$ Dipartimento Energia, Politecnico di Torino, C.so Duca degli Abruzzi 24, 10129 Torino, Italy
}

\begin{abstract}
We compute the classical eddy current losses in Soft Magnetic Composite (SMC) materials, taking into account the eddy current paths appearing at the scale of the sample cross-section because of random contacts between the grains. The prediction of this loss contribution is a challenging task, because of the stochastic nature of the associated conduction process. We start our study from an identification of the statistical properties of the contacts between grains, starting from resistivity measurements. We then develop a numerical loss model for random grain-to-grain conduction, by which we demonstrate that the classical loss in SMCs can be decomposed into a contribution deriving from the eddy currents circulating inside the grains and a contribution due to the macroscopic eddy currents flowing from grain to grain via random contacts. An experimental validation of this model is proposed for a representative SMC material, where the magnetic losses are measured in ring samples with a range of cross-sectional areas.
\end{abstract}

\section{Index Terms -Soft Magnetic Composites (SMC), Eddy current losses, Classical losses, Conductivity of heterogeneous materials}

56 resistivity $\rho_{\text {meas }}$, which can be seen as an intrinsic and directly 57 accessible property of the material. The results presented here 58 show that this purpose can be achieved by properly taking into 59 account the random nature of the contacts between grains.

60

61

75

76

\begin{tabular}{ccc}
\hline \multicolumn{3}{c}{ MEASURED AND COMPUTED RESISTIVITY FOR THE TWO TOROIDS } \\
\hline Toroid & $\rho_{\text {meas }}(\mu \Omega \cdot \mathrm{m})$ & $\hat{\rho}(m, s)(\mu \Omega \cdot \mathrm{m})$ \\
\hline 1 & 1020 & 930 \\
2 & 850 & 897 \\
\hline
\end{tabular}

\section{3D RANDOM CONTACT RESISTIVITY MODELING AND IDENTIFICATION}

We have investigated commercial SMCs by Höganäs [11]. Resistivity measurements have been carried out, for a given material, in ring samples of different square cross-sectional areas $6.25 \mathrm{~mm}^{2}$ (toroid 1) and $25 \mathrm{~mm}^{2}$ (toroid 2). From micrographic analysis we get the average side length of the grains (assumed to be square) $l_{\mathrm{g}}=114 \mu \mathrm{m}$. See [12] for an example of micrograph in such materials.

The resistivities of the two toroids $\rho_{\text {meas }}{ }^{(1)}$ and $\rho_{\text {meas }}{ }^{(2)}$ have been measured using an indirect method, discussed in [13][12], where the ring sample is made to load the secondary circuit of a transformer. The results are shown in Table I.

TABLE I

MEASURED AND COMPUTED RESISTIVITY FOR THE TWO TOROIDS

The resistivity is seen to decrease slightly with increasing the cross-sectional area $S$ of the ring sample. A possible explanation could be that, because of random contacts between grains, the larger is $S$ the lower is the probability of having a current path broken by lack of contact. Let us therefore describe the material granular structure with a regular 3-dimensional distribution (Fig. 1) of cubic grains, where the grain-to-grain contact resistance has randomly distributed values. It results into a network resistance model, where the particles have a node at their center, connected to the neighboring nodes by six contact resistances. The resistances values are assumed to be statistically independent and to follow a lognormal law of parameters $(m, s)$ [14]. A given voltage difference is applied between two parallel 
90 sample cross-sections and the equivalent resistivity offered by 91 the network is calculated. This can be done by numerically 92 inverting the system of equations obtained from the 93 application of Kirchhoff's laws to each node and branch of the 94 resistance network, in order to get the currents in each branch. 95 Then the equivalent resistance is obtained doing the ratio 96 between the applied voltage and the total current absorbed by 97 the network. Knowing the section and the length of the 98 sample, the resistivity can be deduced.

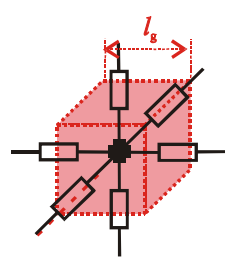

Equivalent cubic particle

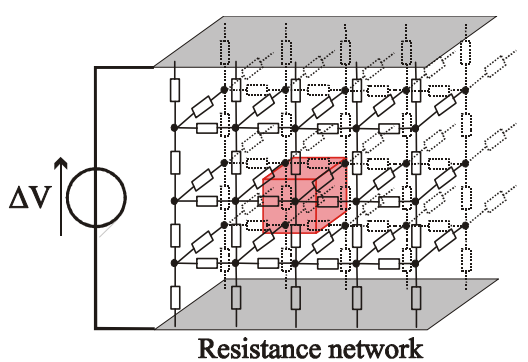

Resistance network
Fig. 1: Modeling the resistive properties of the material by a threedimensional equivalent resistance network.

The optimal statistical parameters $(m, s)$ of the distribution are identified by an optimization algorithm minimizing the quadratic distance (QD) between the relative measured and calculated resistivities for the two considered $S$ values:

$\min _{(m, s)}(\mathrm{QD})=\min _{(m, s)}\left(\frac{\rho_{\text {mes }}{ }^{(1)}-\hat{\rho}^{(1)}(m, s)}{\rho_{\text {mes }}{ }^{(1)}}\right)^{2}+\left(\frac{\rho_{\text {mes }}{ }^{(2)}-\hat{\rho}^{(2)}(m, s)}{\rho_{\text {mes }}{ }^{(2)}}\right)^{2}$

108 The values $m=3.07$ and $s=3.29$ were found to minimize (1) to less than $1.6 \%$. The measured $\left(\rho_{\text {meas }}^{(1)}\right.$ and $\left.\rho_{\text {meas }}^{(2)}\right)$ and predicted $\left(\hat{\rho}^{(1)}\right.$ and $\hat{\rho}^{(2)}$ ) values of the toroids resistivity are reported in Table I. The measured and computed resistivity values are in quite good agreement for the two samples. The discrepancy can be explained by an uncertainty on the measured resistivity values (estimated around 5\%), due to the indirect measurement method based on the induction of eddy currents [13].

\section{NUMERICAL LOSS PREDICTION}

We turn now our attention to the eddy current losses, by developing a bidimensional numerical loss model upon the sample cross-section (see Fig. 2). We assume square iron grains of resistivity $\rho_{\mathrm{i}}=1.04 \cdot 10^{-7} \Omega \cdot \mathrm{m}$ and side length $l_{\mathrm{g}}=$ $114 \mu \mathrm{m}$. The resistances between grains are modeled by resistive layers of infinitely small thickness and their values follow the previously introduced log-normal distribution of parameters $(m, s)$. The skin effect is neglected.

\section{A. Problem formulation}

The equation on the electric vector potential $T$ to compute eddy currents, written in the phasorial form, is the following one:

$$
\left\{\begin{array}{l}
\vec{\nabla} \cdot(\rho \vec{\nabla} T)=i 2 \pi f B_{m} \\
T=0 \text { on the boundary }
\end{array}\right.
$$

130 being $B_{\mathrm{m}}$ the amplitude of the applied sinusoidal induction

131 with frequency $f$ on the cross-section, and $\rho$ the total resistivity

132 of the medium, which is not uniform due to the grain

133 boundary layer presence.

134 Equation (2) is discretized by using the cell method 135 [15][16], where square cells are applied for meshing the toroid 136 cross-section. The one-component current potentials are 137 assigned to the nodes of this primal mesh, whereas the 138 electrical current intensities belong to the primal edges. Under 139 this assumption, the currents are expressed by the relation:

$$
\{j\}=G \cdot\{T\}
$$

140 where $G$ is the edge-node connection matrix. A second, dual,

141

142

143

144 mesh is then superposed to the former (see Fig. 2). Here, the magnetic fluxes belong to the dual surfaces, whereas the electrical voltages are naturally assigned to the dual edges. The Faraday law can then be expressed as:

$$
G^{\mathrm{T}} \cdot\{u\}=-i \cdot 2 \pi f \cdot B_{\mathrm{m}} \cdot h^{2} \cdot\left\{1_{N}\right\}
$$

where $\{u\}$ is the vector of voltages, $f$ is the frequency, $B_{\mathrm{m}}$ is the average induction amplitude in the cross-section, $h$ is the mesh size, and $\left\{1_{N}\right\}$ is the unity vector of size $N$. This size is equal to the number of dual surfaces, i.e. the number of primal nodes not belonging to the boundary. The constitutive law is expressed as:

$$
\{u\}=R(w) \cdot\{j\}
$$

1 where the diagonal matrix $R(w)$, containing the resistances between primal nodes, is partially random because each time a layer between grains is encountered, the random resistance of the corresponding layer portion is added to the one of pure iron. Taking into account (3), (4), and (5), we eventually obtain:

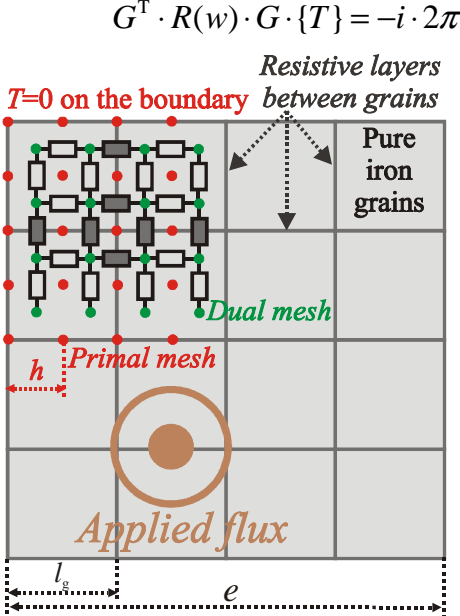

Fig. 2: 2D loss model with random contacts between grains modeled as resistive layers.

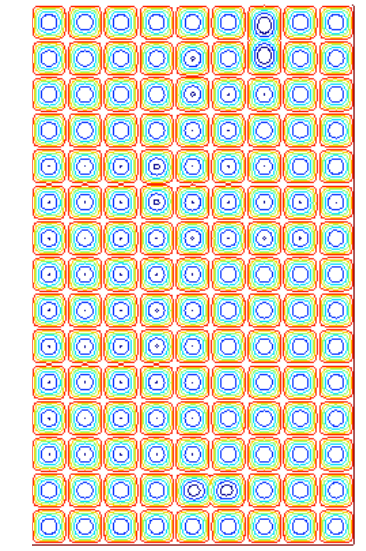

Fig. 3: Example of current paths obtained from numerical simulation $(m=3.07, s=3.29)$.
157

158

159 160
The current potential $\{T\}$ is assumed zero on the boundary to satisfy boundary conditions (2). An example of computation of current paths is given in Fig. 3. The specific classical loss per cycle is obtained by computing the following sum: 


$$
W_{\text {class }}=i \pi B_{m} \frac{1}{N}\left\{1_{N}\right\}^{T}\{T\}
$$

161

162

163

164

165

166

167

168

169

170

171

172

173

174

175

176
The mesh resolution must satisfy a priori the condition $h<<l_{\mathrm{g}}$, in order to correctly provide both the loss inside the grains, and the eddy currents between the cells. This point is discussed in detail in the following sections.

\section{B. Numerical validation}

The proposed model can be validated by numerical experiments, using models already presented in the literature [17]. The authors of this reference have developed a multiscale finite element model able to handle heterogeneous materials, with random resistances between grains. It takes into account the contact thickness and geometry and possible skin effects. The use of a multiscale approach avoids solving the whole problem in a single calculation, considerably limiting the unknown number. The relative magnetic permeability $\mu_{\mathrm{r}}$ is assumed uniform over the cross-section (a value of $\mu_{\mathrm{r}}=400$ is reasonable for commercial SMCs [11]).

The two models have been applied to the computation of the specific classical loss over the toroid cross-section, ( toroid 1 , with number of cells $N=22 \times 22$ ). An average induction of amplitude $B_{\mathrm{m}}=1 \mathrm{~T}$ is considered. The size of the cell $l_{\mathrm{g}}$ is 114 $\mu \mathrm{m}$ (for the model [17], an average contact thickness, lower than $1 \mu \mathrm{m}$ has been chosen, based on the density considerations made in [10]). The statistical contact parameters $(m, s)$ are those derived in the previous section. The results are shown in Fig. 4. The two models provide similar results, thereby validating the previous approach.

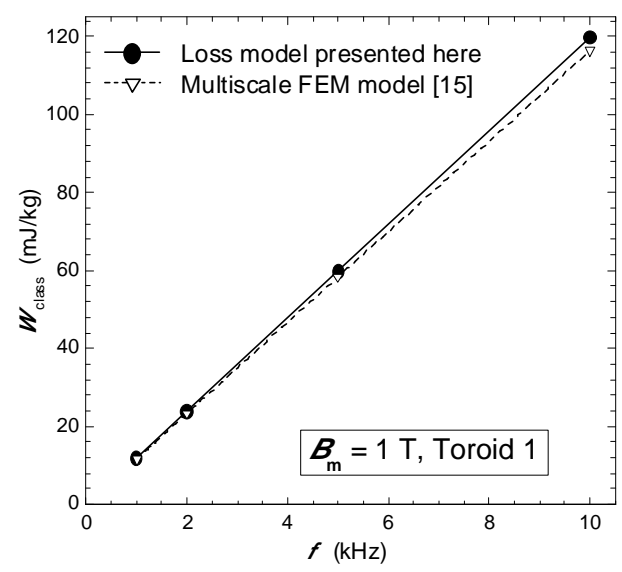

Fig. 4: Classical loss in function of frequency $\left(B_{\mathrm{m}}=1 \mathrm{~T}\right)$ for the toroid 1 , obtained with the cell model (neglecting skin effect) and with the multiscale FEM model [17].

It is noted that the skin effect is negligible, which results in a linear dependence of the classical loss versus frequency. This fact has been checked experimentally in [10], and is confirmed here by numerical simulation. This means that the eddy currents are not high enough to change locally the induction. Thus, all the local loss components (hysteresis, excess loss) are not affected by the presence of eddy currents. It is shown in the next section that it is possible to decompose the classical loss under two components: a "microscopic" part, restricted to the scale of the particles, and a "macroscopic" one, associated with the long-range eddy currents.

\section{Loss decomposition}

Since skin effect can be disregarded, the classical loss at the scale of the particle can be calculated independently of the classical loss associated with the macroscopic scale. We therefore write:

$$
W_{\text {class }}=W_{\text {classMIC }}+W_{\text {classMAC }}
$$

To check this equality, the specific microscopic loss term $W_{\text {class MIC }}$ is computed by a calculation over a single cell of pure iron. The macroscopic classical term $W_{\text {classMAC }}$ is computed by using only one cell per particle (i.e. $h=l_{\mathrm{g}}$ ). In this way, only the loss due to the current flowing from grain to grain is obtained. This loss can be compared with the full classical loss $W_{\text {class }}$ obtained by imposing an important number of cells per grain $\left(h<<l_{\mathrm{g}}\right)$, as done in the previous section. The results are shown in Fig. 5, for the toroid 1 and a sinusoidal applied induction $B_{\mathrm{m}}=1 \mathrm{~T}$. A significant macroscopic loss contribution can be observed for example for $m=0.5$ and $s=0.25$. It is obvious that the classical loss decomposition method seems to provide good results, although it is not straightforward from a mathematical point of view.

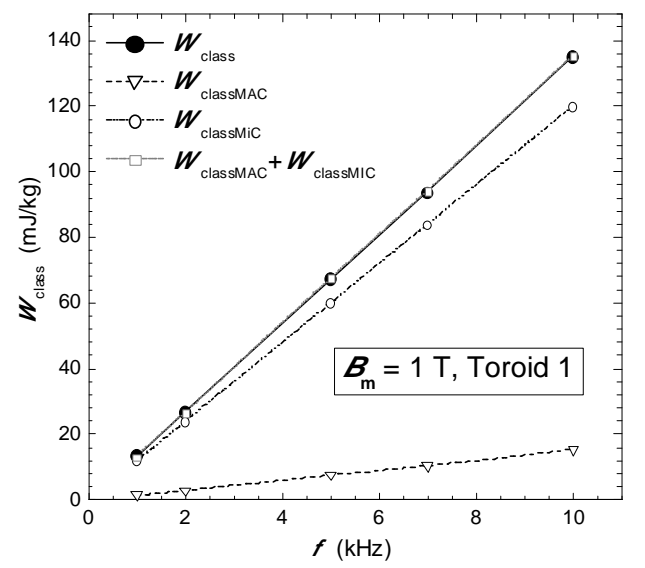

Fig. 5: Classical loss versus frequency $\left(B_{\mathrm{m}}=1 \mathrm{~T}\right)$ for the toroid 1, obtained either directly by the classical loss model or by summing the macroscopic and microscopic terms $(m=0.5$ and $s=0.25)$

This behavior can be explained taking into account that it is always possible, for a given point of a given cell, to separate the eddy current density as $\vec{J}=\vec{J}_{M I C}+\vec{J}_{M A C}$, where $\vec{J}_{M I C}$ is restricted at the scale of the grain (i.e. its volume integral on the grain is zero), and another component $\vec{J}_{M A C}$ whose 32 integral on the grain is different from zero (Fig. 6).

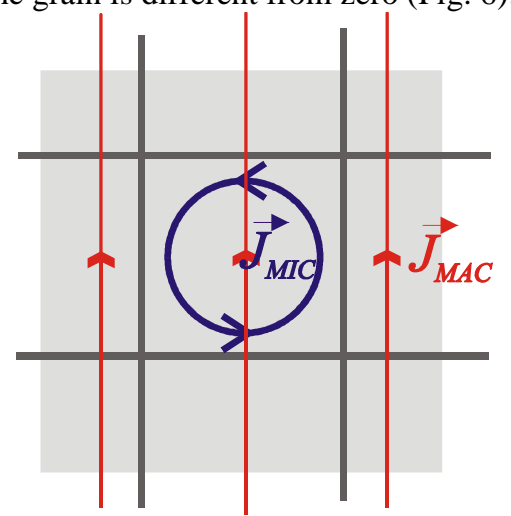

Fig. 6: Eddy current decomposition between a microscopic and macroscopic eddy current component 
Then, the classical loss is obtained as:

$$
W_{\text {class }}=\frac{1}{f} \int_{S} \rho \vec{J}^{2} d S=\frac{1}{f} \sum_{N} \int_{S_{\text {cell }}} \rho\left(\vec{J}_{M I C}+\vec{J}_{M A C}\right)^{2} d S
$$

$$
W_{\text {class }}=\frac{1}{f} \sum_{N_{\text {cell }}}\left(\int_{S_{\text {cell }}} \rho \vec{J}_{M A C}^{2} d S+\rho_{i} \int_{S_{\text {cell }}} \vec{J}_{M I C}^{2} d S+\rho_{i} \int_{S_{\text {cell }}} \vec{J}_{M A C} \cdot \vec{J}_{M I C} d S\right)
$$

If the number of cells is important, which is always the case for a heterogeneous material, the macroscopic eddy current component can be considered as uniform at the scale of the grain. Thus the integral of the last term is zero, because the integral of the microscopic term over the cell is zero. The first integral is the macroscopic classical loss component, while the second one represents the microscopic contribution. This calculation gives a theoretical justification to the decomposition proposed in (8).

Thus, the classical loss can be decomposed into two components (i.e. a microscopic and macroscopic one) provided that:

- The skin effect is negligible, in such a way that the macroscopic term does not change the local induction distribution.

- The macroscopic term can be considered uniform at the scale of the single grain, so that the cross product contribution in (10) vanishes.

\section{Experimental validation of the model}

In absence of skin effect, the specific loss terms at the scale of the particle are the same (hysteresis, excess, and microscopic eddy currents) for the two samples. Thus, the loss difference between the two toroids 1 and 2 for the same $B_{\mathrm{m}}$ and frequency $f$ is only due to the difference of macroscopic loss terms:

$$
\Delta W=W^{(2)}-W^{(1)}=W_{\text {classMAC }}{ }^{(2)}-W_{\text {classMAC }}{ }^{(1)}
$$

This loss difference is computed by the previous random model and compared with experiments. The results are given in Fig. 7, for a peak induction $B_{\mathrm{m}}=1 \mathrm{~T}$, with the parameters $m$ $=3.07$ and $s=3.29$ for the lognormal law, identified from resistivity measurements, have been adopted. The results obtained with the plain literature model [6], taking the mean measured resistivity of Table I $\left(\rho_{\text {meas }} \approx 913 \mu \Omega \cdot \mathrm{m}\right)$, and assuming an homogeneous material of the same resistivity for the computation of the macroscopic eddy currents paths are also reported. It is apparent the gross disagreement of such results with the experiments, being the predicted loss figure $\Delta W$ about three times higher than the experimental one.

\section{CONCLUSION}

A model taking into account the random contacts between grains is necessary for a correct loss prediction in SMCs. The validity of an approach based on the decomposition of the classical loss in heterogeneous material into a macroscopic and local eddy current term, has been demonstrated. This
285 correctly accounts for the experiments carried out in toroidal SMC samples of different sizes.

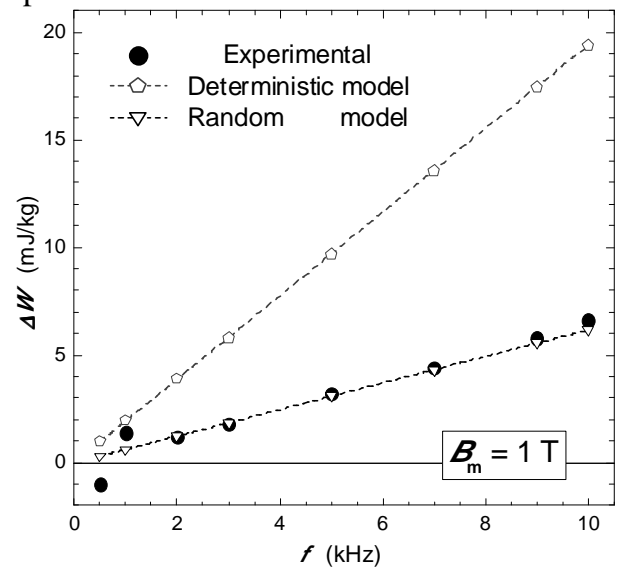

Fig. 7: Energy loss difference $\Delta W\left(B_{\mathrm{m}}=1 \mathrm{~T}, f\right)$ between toroids 1 and 2, experimentally and by the classical loss model (random and deterministic)
290

\section{REFERENCES}

[1] J. Cros, P. Viarouge, and M.T. Kakhki, "Design and Optimization of Soft Magnetic Composite Machines With Finite Element Methods," IEEE Trans. Magn., vol. 47, no. 10, pp. 4384--4390, 2011.

[2] F. Marignetti, V.D. Colli, and S. Carbone, "Comparison of axial flux PM synchronous machines with different rotor back cores," IEEE Trans. Magn., vol. 46, no. 2, pp. 598-601, 2010.

[3] H. Vansompel, P. Sergeant, and L. Dupré, "Optimized design considering the mass influence of an axial flux permanent-magnet synchronous generator with concentrated pole windings," IEEE Trans. Magn., vol. 46, no. 12, pp. 4101-4107, 2010.

[4] L. Li, A. Kedous-Lebouc, A. Foggia, and J.C. Mipo, "Influence of magnetic materials on claw pole machines behavior," IEEE Trans. Magn., vol. 46, no. 2, pp. 574-577, 2010.

[5] A. Chebak, P. Viarouge, and J. Cros, "Analytical Computation of the Full Load Magnetic Losses in the Soft Magnetic Composite Stator of High-Speed Slotless Permanent Magnet Machines," IEEE Trans. Magn., vol. 45, no. 3, pp. 952-955, 2009.

[6] M. Anhalt and B Weidenfeller, "Dynamic losses in FeSi filled polymer bonded soft magnetic composites," J. Magn. Magn. Mater., vol. 304, no. 2, pp. e549-e551, 2006.

[7] A.H. Taghvaei, H. Shokrollahi, K. Janghorban, and H. Abiri, "Eddy current and total power loss separation in the iron-phosphatepolyepoxy soft magnetic composites," Materials and Design, vol. 30, no. 10, pp. $3989-3995,2009$.

[8] O. Bottauscio, A. Manzin, V.C. Piat, M. Codegone, and M. Chiampi, "Electromagnetic phenomena in heterogeneous media: Effective properties and local behavior," J. Appl. Phys., vol. 100, p. 044902, 2006.

[9] C. Yanhong and GB. Kliman, "Modeling of soft magnetic composites," in IEEE Industry Applications Conference (IAS), 2004.

[10] A. Bordianu, O de la Barrière, O. Bottauscio, M. Chiampi, and A. Manzin, "A Multiscale Approach to Predict Classical Losses in Soft Magnetic Composites," IEEE Trans. Magn., vol. 45, no. 3, pp. 952-955, 2012. [11] Höganäs SMC Brochures. [Online]. http://hoganas.com/en/Products--Applications/Soft-MagneticComposites/SMC-Brochures-Pics/

[12] O de la Barrière, C. Appino, F. Fiorillo, C. Ragusa, H. Ben Ahmed, M. Gabsi, F. Mazaleyrat, M. LoBue, "Loss separation in soft magnetic composites", J. Appl. Phys., vol. 109, p. 07A317, 2011. measurement on soft magnetic composite materials," Przeglad Elektrotechniczny, vol. 83, no. 4, pp. 103-104, 2007.

[14] S. Ross, Probality and Statistics for Engineers and Scientists, 4th ed., Academic Press: Elsevier, 2009, pp. 239.

E. Tonti, "A direct discrete formulation of field laws: The cell method," CMES-Comput. Model. Eng. Sci., vol. 2, no. 2, pp. 237-258, 2001. [16] [Online]. Available: http://discretephysics.dica.units.it/

for the Analysis of Fine Periodic Electromagnetic Structures," in Compumag, Sydney, 2011. 